http://www.inass.org/

\title{
Effects of Network Topology on Decision-Making Behavior in A Biological Network Model
}

\author{
Suojun Lu*, Jian'an Fang, Qingying Miao \\ College of Information Science \& Technology, Donghua University, \\ 2999 North Renmin Road, Songjiang District, Shanghai 201620, China. \\ * Corresponding author’s E-mail: lusjun@mail.dhu.edu.cn
}

\begin{abstract}
The topology of a network often plays a crucial role in determining its dynamical features. There is increasing interest in trying to understand the relationship between the structural properties of networks and their behaviors. However, this problem has not been fully considered in many neural networks, which mimic certain biological processes. Here, we construct a recurrent network model to simulate the decision-making process of brain. In this model, we examined the effects of network topology on the performance of decision-making by constructing three different topological networks: the regular network, the random network, and the small-world network. We found that the regular network and the small-world network show significant better performance of decision-making than that in the random network when the internal noise of the networks is low. However, following the increase of the internal noise, the random network, instead of two other networks, shows better ability to resist the noise. Finally, to mimic neurodegeneration or neural injury, we introduced two types of neuronal damages: clustered damage and distributed damage. We found that three networks exhibit different network behaviors in the case of neuronal damages. The regular network and the small-world network display severe decrease of the performance in the distributed damage pattern, but not in the clustered damage pattern. The random network shows similarly gradual decrease of the performance in both damage patterns. Furthermore, the small-world network shows the best performance in the high levels of distributed damage. Together, our results indicate that network topology significantly influence the network behaviors in our model of decision-making.
\end{abstract}

Keywords: complex network; small-world; decision-making; recurrent model.

\section{Introduction}

Decision-making is an essential cognitive behavior for daily life in animals or even in human being (for reviews, see Schall, 2001; Platt, 2002; Glimcher, 2003; Smith \& Ratcliff, 2004; Sugrue et al., 2005). By applying physiological and psychophysical techniques, neuroscientists have obtained many experimental data, which offer the basis for further theoretical analysis. For example, in a visual motion discrimination task, the monkey is trained to make a judgment about the direction of motion in a near-threshold stochastic random dot display and to report the perceived direction with a saccadic eye movement. Electrophysiological recording as well as functional brain imaging has been carried out to link the animal's behavior to neural activities in specific brain areas (Newsome et al., 1989; Britten et al., 1993; Parker \& Newsome, 1998; Romo \& Salinas, 2001; Platt, 2002; Roitman \& Shadlen, 2002; Glimcher, 2003; Romo \& Salinas, 2003). Based on the biological findings, some computational models have been established to estimate and interpret the decision-making processes of brain (Vickers, 1970; Busemeyer \& Townsend, 1993; Usher \& McClelland, 2001; Wang, 2002; Mazurek et al., 2003; Bogacz et al., 2006; Ditterich, 2006; Wong \& Wang, 2006; Bogacz, 2007).

A recurrent network model shows that slow synaptic reverberation as well as winner-take-all competition can generate attractor dynamics that reproduce both neurophysiological and psychophysical data (Wang, 2002). In this model, each neuron receives input from all other neurons, and "Hebbian" rule (the synapse is strong or weak if 
in the past two cells tended to be active in a correlated or anti-correlated manner) has been used to generate the synaptic weights. The author did not explore the effects of network topology on the decision-making process. However, recent advances in the field of complex network show that different network architectures have different network properties and generate different network behaviors (for reviews, see Albert \& Barabasi, 2002; Newman, 2003; Boccaletti et al., 2006). Instead that the connection topology is assumed to be either completely regular or completely random, many biological, technological and social networks lie somewhere between these two extremes. Based on the findings that many systems are highly clustered, like regular lattices, yet have small characteristic path lengths, like random graphs, Watts and Strongatz proposed a "small-world" network (Watts \& Strogatz, 1998). They also found that small-world network displays enhanced signal-propagation speed, computational power, and synchronizability. These properties may contribute to the computational process in choosing alternative signals. Therefore, here we investigate the effects of network topology on the decision-making process, corresponding to the visual motion discrimination experiments.

By constructing three different topological networks (the regular network, the random network, and the small-world network), we found that the regular component of network architecture improves the performance in decision-making process when the internal noise of the networks is low. However, the networks with high random component display better performance in the high level of the internal noise. Furthermore, different networks also show different changes of network behaviors in the case of neuronal damages. Our results indicate that the small-world network, which is endowed with both advantages of the regular network and the random network, displays the best performance in the decision-making process after neuronal damages, especially in the distributed damage pattern.

\section{Model}

As shown in Figure 1, there are two neural groups, each of which is sensitive to one of the two stimuli ( $I_{1}$ and $I_{2}$, mimic the motion to left or to right), compete with each other through self-recurrent $\left(\mathrm{w}^{+}\right)$and mutual inhibition (w-). In addition, Gaussian distributed noise (NS) was introduced to the networks to mimic the spontaneous neuronal activities (as the internal noise). Inside the neural groups, neurons are connected with each other in different topological manners to form three types of networks: the regular network, the random network, and the small-world network. Here the scheme only shows the small-world network (black links for regular components, white links for random components).

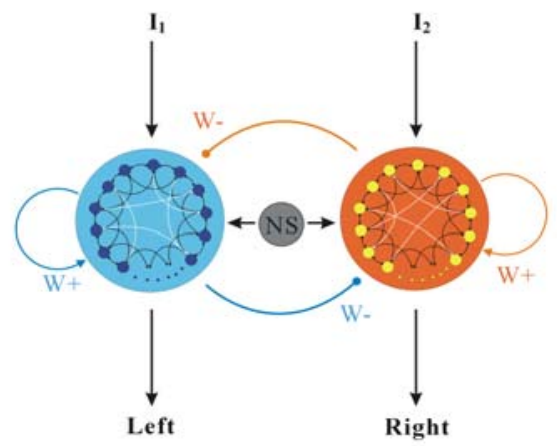

Figure 1 Schematic of the model architecture in the decision-making process.

We used a recurrent network model (Amit \& Brunel, 1997; Hansel et al., 1998; Durstewitz et al., 2000; Wang, 2002) with two competing neural groups ( $N=200$ for each group) to simulate the process of decision-making in the visual motion discrimination task (Fig. 1). Each neuron receives positive input from neurons within the same group by certain network connection, and receives negative input from every neuron within the alternative group. We used three different topological networks to construct the network architecture within each neural group: the regular network, the random network, and the small-world network. For the regular network, each neuron is adjacently connected with $k / 2(k=20)$ leftward neurons and $k / 2$ rightward neurons to form a circuit loop. For the random network, each neuron is randomly connected with other neurons with the inward degree $k_{\text {in }}\left(k_{\text {in }}=20\right)$ and outward degree $k_{\text {out }}\left(k_{\text {out }}=20\right)$. For the small-world network, neuronal connections are modified from the regular network described above with Watts and Strogtz's method (Watts \& Strogatz, 1998). That is, parts of regular connections $(10 \%)$ are randomly reconnected to keep the final degrees constant $\left(k_{i n}=\right.$ $\left.20, k_{\text {out }}=20\right)$. All connections mentioned above are unidirectional. However, due to the symmetrical structure of the regular network, eventually neurons show reciprocal connections with each other in the parts of network connections which are composed of the regular component. The statistical results of network topology are shown in Table 2. 
Model neurons (i) are described by their total synaptic input current $\mathrm{I} i$, which evolves in time according to the leaky-integrator differential equation:

$\tau$ SydI $\left._{i} / d t=-I_{i}+\sum w_{i j} R\left(I_{j}\right)-G \sum R R\left(I_{j}\right)\right]+I_{a f f}+I_{b k}$,

where $\tau$ sy is the integration time constant of the synapse $(\tau s y=50 \mathrm{~ms}), w_{i j}$ is the strength of the synaptic connection from unit $j$ to unit $i\left(w_{i j}=0\right.$ or 1), $R\left(I_{j}\right)$ is the firing rate of neuron $j, G$ is the inhibition function from alternative neural group, $I_{\text {aff }}$ is the afferent input (or external stimuli), and $I_{b k}$ is the internal noise. The firing rate of a neuron is assumed to be:

$$
\begin{aligned}
& R\left(I_{i}\right)=0, \\
& \text { if } I_{i}<\theta_{\text {exc }} \\
& R\left(I_{i}\right)=\alpha \ln \left(I_{i} / \theta_{\text {exc }}\right), \quad \text { else if } \theta_{\text {exc }} \leq I_{i}<\theta_{\text {max }} \text {, } \\
& R\left(I_{i}\right)=\alpha \ln \left(\theta_{\max } / \theta_{\text {exc }}\right), \quad \text { else } I_{i} \geq \theta_{\max }
\end{aligned}
$$

where $\theta_{\text {exc }}$ is lower firing threshold ( $\theta_{\text {exc }}=0.6$ ), $\theta_{\max }$ is the upper threshold of neuronal firing rate $\left(\theta_{\max }=3.0\right), \alpha$ is a free parameter of firing rate $(\alpha e x=0.5)$. The inhibition function $G$ is assumed to be a linear function:

$$
G(x)=\beta x,
$$

where $\beta$ is a free parameter of the inhibition strength $(\beta=0.1)$. The synaptic transmission is simply simulated with a constant delay ( $d s y=4 \mathrm{~ms}$ ).

The internal noise $I_{b k}$ is introduced to mimic the spontaneous activities of neurons, which is described as the following equation:

$$
d I_{b k} / d t=-\left(I_{b k}-I_{0}\right) / \tau n s+\sigma n(t),
$$

where $\tau n s$ is the integration time constant of the noise ( $\tau n s=5 \mathrm{~ms}), I_{0}$ is the initial state of synaptic current $\left(I_{0}=0.1\right), n(t)$ is a white-noise with zero mean and unit standard deviation, and $\sigma$ is the modifiable factor of the white-noise, which corresponds to the virtual standard deviation of the noise. For most of the simulations, we used

\begin{tabular}{|c|c|c|}
\hline $\mathrm{k}_{\text {in }}$ & Inward degree of each node & 20 \\
\hline $\mathrm{k}_{\text {out }}$ & Outward degree of each node & 20 \\
\hline $\mathrm{T}_{\text {total }}$ & Running Time for each trial (ms) & 800 \\
\hline $\mathrm{dt}$ & Integration of time step (ms) & 0.4 \\
\hline $\mathrm{p}_{\mathrm{st}}$ & Proportion of the stimulated neurons & 0.3 \\
\hline$\mu_{0}$ & the basal level of the stimuli & 0.25 \\
\hline$c^{\prime}$ & coherent level of the stimuli & variable \\
\hline$\theta_{\mathrm{exc}}$ & $\begin{array}{l}\text { lower firing threshold for synaptic } \\
\text { transmission }\end{array}$ & 0.6 \\
\hline$\theta_{\max }$ & upper firing threshold & 3 \\
\hline$\theta_{\text {out }}$ & $\begin{array}{l}\text { threshold of the current to generate } \\
\text { behavior }\end{array}$ & 1 \\
\hline$p_{\text {out }}$ & $\begin{array}{l}\text { threshold of the proportion to generate } \\
\text { behavior }\end{array}$ & 0.6 \\
\hline$\tau_{\mathrm{sy}}$ & time constant of synapse (ms) & 50 \\
\hline$d_{\text {sy }}$ & $\begin{array}{l}\text { delay time of synaptic transmission } \\
\text { (ms) }\end{array}$ & 4 \\
\hline$\alpha$ & a free parameter of firing rate & 0.5 \\
\hline$\beta$ & a free parameter of inhibition strength & 0.1 \\
\hline$\tau_{\mathrm{ns}}$ & time constant of noise (ms) & 5 \\
\hline$\sigma$ & $\begin{array}{l}\text { standard deviation of the internal } \\
\text { noise }\end{array}$ & variable \\
\hline
\end{tabular}
$\sigma=0.5$. To examine the effects of the internal noise on the network behaviors, we varied $\sigma$ from 0.5 to 1.0 .

Table 1 Range of model parameters.

\begin{tabular}{lll}
\hline Parameters & Description & Value \\
\hline $\mathrm{N}$ & Neuron number within each group & 200
\end{tabular}

Table 2 Statistics of topological features in three networks: inward degree $\left(k_{\text {in }}\right)$, outward degree $\left(k_{\text {out }}\right)$, cluster coefficient $(c c)$, and path length $(p l)$. (SW indicates small-world).

\begin{tabular}{ccccc}
\hline Network & $\mathrm{K}_{\text {in }}$ & $\mathrm{K}_{\text {out }}$ & $\mathrm{cc}$ & $\mathrm{pl}$ \\
\hline regular & 20 & 20 & 0.711 & 5.477 \\
random & 20 & 20 & 0.191 & 2.02 \\
$\mathrm{SW}$ & 20 & 20 & 0.502 & 2.436 \\
\hline
\end{tabular}

\section{Results}

In this section, we investigate the effects of network topology on the decision-making process in three different aspects. The first part of this section examines the effects of network topology on the performance of decision-making. The second part examines the effects of the internal noise on the decision-making process. The third part examines the effects of neuron damages (including the 
clustered damage pattern and the distributed damage pattern) on the decision-making process in three different topological networks.

\subsection{Effects of network topology on decision making process}

After a short delay period (200 ms), two stimuli $\mu_{A}$ and $\mu_{B}$ are delivered to the parts of two neural groups ( $p s t=30 \%$ ) respectively with duration of $400 \mathrm{~ms}$. Then another delay period (200 $\mathrm{ms}$ ) is introduced, serving for additional time to generate the decision-making process. Therefore, the total simulation time ( $\left.T_{\text {total }}\right)$ is $800 \mathrm{~ms}$. To mimic the visual motion discrimination task in the monkey, we used the input coherent $c^{\prime}$ (from 0 to 1.0 ) to linearly correlate two stimuli $\mu_{A}$ and $\mu_{B}$ :

$$
\begin{aligned}
& \mu_{A}=\mu_{0}\left(1+c^{\prime}\right), \\
& \mu_{B}=\mu_{0}\left(1-c^{\prime}\right)
\end{aligned}
$$

where $\mu_{0}$ is the base level of the stimuli ( $\mu_{0}=0.25$ ). Considering the internal noise, at low coherent (small $c^{\prime}$ ), the stimuli to the two neural groups are similar and hard to distinguish. However, the competition between these two neural groups will eventually lead to one of the two attractor state, in which one neural group displays elevated persistent activity while the other group's activity is suppressed. Suppose that neural group $A$ receives stronger stimuli $\mu_{A}$, while neural group $B$ receives weaker stimuli $\mu_{B}$. For instance, in a trial at the coherent level 0.2, the regular network (Fig. 2 (a)) and the small-work (Fig. 2 (b)) generate robust decision-making process indicated by persistent activity of the group $A$, while the random network (Fig. 2 (c)) fails to generate winner-take-all pattern. The firing rate (or the synaptic current) of the group $\mathrm{A}$ in the random network drops to the base level after the offset of the stimulus.

To quantify the network behaviors, we used the percentage of correct choice and reaction time as two criteria. The choice is considered as a "correct" action if one neural group, which receives higher external stimulus, generates positive persistent activity across certain firing threshold $\theta_{\text {out }}\left(\theta_{\text {out }}=1.0\right)$ with a certain percentage $p_{\text {out }}\left(p_{\text {out }}=0.6\right)$ of activated neuron number. The time point across the threshold is defined as the reaction time. Therefore, high correct choice or low reaction time indicates the good performance of decision-making. As shown in Fig. 2, following the increased coherent level, the percentage of correct choice (Fig. 2 (d)) for all networks is increased while the reaction time (Fig. 2 (e)) is decreased. Moreover, we found that the regular network and the small-world network show significant higher correct choice and lower reaction time than those in the random network, especially at low coherent levels $(0.05-0.2)$. These results indicate that the regular component of the network architecture amplifies the small difference between two similar signals and improves the speed of signal recruitment during the competition of two neural groups.
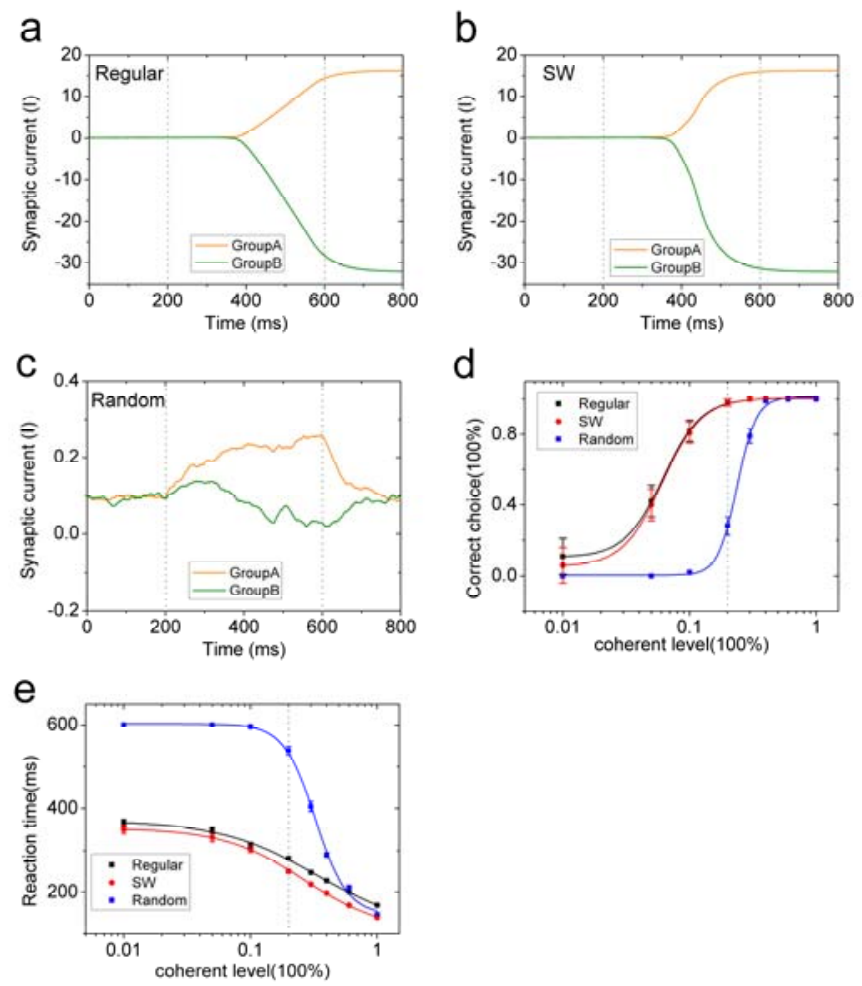

Figure 2 Effects of network topology on the performance of decision-making.

As shown in Figure 2, an individual trial of decision-making process at the coherent level 0.2 in three different topological networks. The curves are the averaged synaptic input currents of the neural group $(N=200)$. The neural group $A$ is supposed to receive stronger stimuli, while the neural group $B$ receives weaker stimuli. Two dotted lines indicate the onset and the offset of the stimuli respectively. The regular network and the small-world network show significantly higher correct choice and lower reaction time than those in the random network. The decision-making process is simulated in the low internal noise $(\sigma=0.5)$. Data are fitted by the 
logistic curve ( $R^{2}>0.99$ for all curves). In each test point, the data are obtained after average of 100 trials. The error bar indicates mean standard error (SEM).

\subsection{Effects of the internal noise on network behaviors}

Biological decision-making process might not be entirely reliable due to the noise in the sensory system or in the environment. As mentioned above, we introduced the internal noise to mimic neuronal spontaneous activities, which may be activated by stochastic background inputs inside the brain, rather than external stimuli. However, it's difficult to determine the appropriate amplitude of the noise, which responds to the physiological state of real neurons. Thus, here we examined the effects of the internal noise on network behaviors by systematically varying the standard deviation $\sigma$ from 0.5 to 1.0. As shown in Fig. 3a, neural networks show spontaneous decision-making, which is considered as incorrect choices, in the condition of high internal noises $(\sigma>0.7)$ even without external stimuli. The percentage of incorrect choice is increased when the standard deviation $\sigma$ is higher. Finally all networks show completely incorrect choices in the noise level $\sigma=1.0$. Further comparison of network behaviors among different topological networks shows that the random network has the best ability to resist the internal noise in a wild range (form $\sigma=0.75$ to $\sigma=0.9$ ), while the regular network and the small-world network have the similar noise-resistant ability (Fig. 3a). For instance in a trial $(\sigma=0.8)$, the regular network (Fig. 3 (b)) and the small-world network (Fig. 3(c)) generate spontaneous decision-making process, while the random network (Fig. 3 (d)) only shows very low level of the firing rate. These results indicate that the random component of the network architecture contributes to the resistance to the internal noise. a
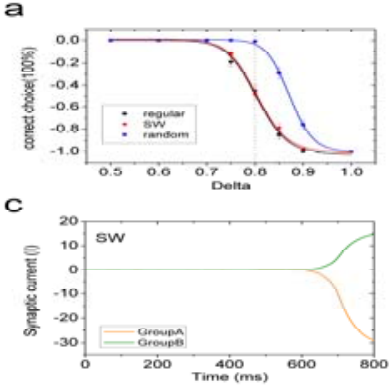

$b$

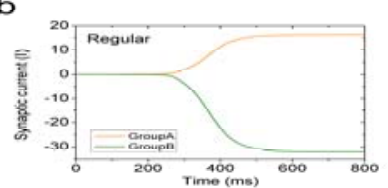

d

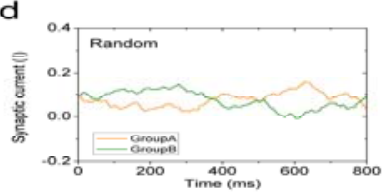

Figure 3 Effects of network topology on the resistance to the internal noise.
As shown in Figure 3, in the high levels of the internal noise (See Fig. 3(a)), the networks generate spontaneous output of decision-making even without the external stimuli, which is considered as a negative correct choice. The random network shows significantly better ability to resist the internal noise. Data are fitted by the logistic curve $\left(R^{2}>0.99\right.$ for all curves). In each test point the data are obtained after average of 100 trials. The error bar indicates SEM. An individual trial of spontaneous decision-making process $(\sigma=0.8)$ in the regular network (Fig. 3(b)), the small-world network (Fig. 3(c)), and the random network (Fig. 3(d)). The curves are the averaged synaptic currents of the neural group $(N=200)$. The neural group $A$ is supposed to receive stronger stimuli, while the neural group $B$ receives weaker stimuli.

To further investigate the effects of the internal noise on the decision-making process, we compared the network behaviors in the presence of both external stimuli and high internal noise. As shown in Figure 4(a), the random network shows the best performance of correct choice in the condition of high internal noise $(\sigma=0.7)$ instead of the worst performance in the condition of low internal noise $(\sigma=0.5)$. The correct choice of the small-world network is similar to the performance of the regular network. However, the random network still shows the worst performance in the aspect of reaction time (Fig. 4(b)). Furthermore, the small-world networks exhibit slightly lower reaction time even than that of the regular network at the whole range of stimuli. These data confirm the results that the regular component improves the speed of signal recruitment while the random component contributes to the resistance of the internal noise. a

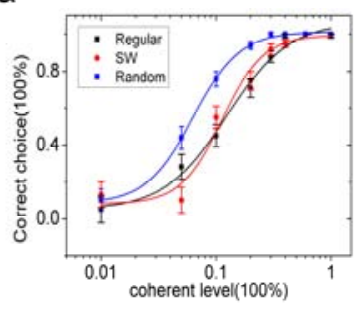

b

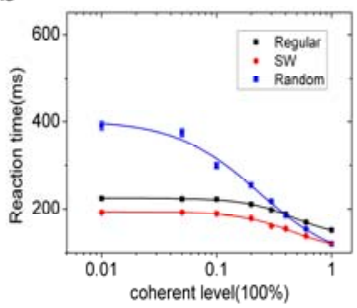

Figure 4 Effects of network topology on the performance of decision-making in the high internal noise.

The random network shows significantly higher correct choice than that in the regular network and the small-world network (a), while the small-world network show significantly lower reaction time than 
that in two other networks (b). The decision-making process is simulated in the high internal noise $(\sigma=0.7)$. Data are fitted by the logistic curve ( $R^{2}>0.97$ for all curves). In each test point the data are obtained after average of 200 trials. The error bar indicates SEM.

\subsection{Effects of neuronal damage on network behaviors}

The nervous system may be damaged by some physical or biological processes, such as mechanical neural injure or neurodegenerative disease. Here we mimic these neuronal damages to examine the network behaviors of decision-making process in different topological networks. Due to the network architectures, different damage patterns may have different effects on the decision-making process. Therefore, we introduced two kinds of damage patterns to the networks: the clustered damage pattern and the distributed damage pattern. The clustered damage pattern refers to the damage of adjacent neurons from $i$ to $i+n$, where $\mathrm{n}$ indicates the number of damaged neurons. The distributed damage pattern refers to random damage of neurons inside a neural group.

We first examined the effects of clustered damage on the network behaviors. As shown in Fig. 5(a), the regular network shows similar correct choice at different levels of clustered neuronal damages. The reaction time of the regular network in the lower coherent level is slightly decreased under the conditions of neuronal damages (Fig. 5 (b)). On the contrary, the random network shows gradually decreased correct choice under the damage percentage $\left(p_{\text {dam }}\right)$ from 0.2 to 0.6 (Fig. $5(\mathrm{c}))$. In addition, the reaction time of the random network is also gradually increased, following the increase of $p_{\text {dam }}$ (Fig. 5 (d)). Finally, the small-world network shows unaffected correct choice at the levels of $p_{\text {dam }}$ from 0.2 to 0.4 (Fig. 5 (e)). At the level $p_{d a m}=0.6$, the percentage of correct choice in the small-world is slightly decreased. The small-world network also shows gradually increased reaction times, following the increase of damage levels (Fig. 5 (f)). The comparison of network behaviors including both correct choice and reaction time shows that the regular network and small-world network have significantly better performance of decision-making than that in the random network at the level $p_{\text {dam }}=$ 0.6 (Fig. 5 (g) and Fig. 5 (h)). These results indicate that the regular component of network architecture contributes to the ability to resist the clustered neuronal damage.
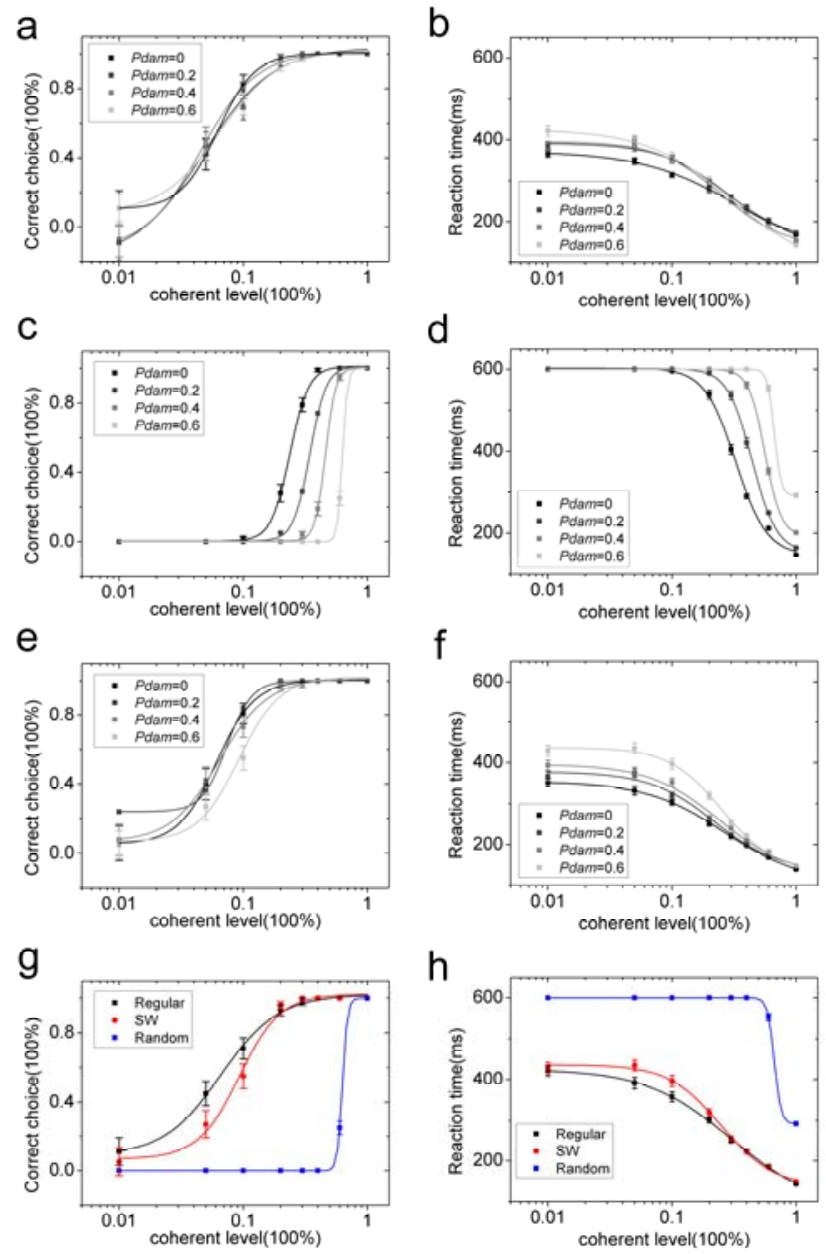

Figure 5 Effects of network topology on the performance of decision-making in the case of the clustered neuronal damage.

As shown in Figures 5(a) and 5(b), the regular network shows slight change of correct choice and reaction time in the decision-making process, following gradual increase of neuronal damages. In Figures 5(c) and 5(d), the random network shows gradual decrease of correct choice and gradual increase of reaction time, following the increase of neuronal damages. In Figures 5(e) and 5(f), the small-world network shows similar change pattern to the regular network. In Figures 5(g) and 5(h), the comparison of network behaviors $\left(p_{\text {dam }}=0.6\right)$ shows that the regular network and the small-world network display significantly better performance than that in the random network. The decision-making process is simulated in the low 
internal noise $(\sigma=0.5)$. Data are fitted by the logistic curve ( $R^{2}>0.98$ for all curves). In each test point the data are obtained after average of 100 trials. The error bar indicates SEM.

Then, we examined the effects of distributed damage on the network behaviors. Unlike the clustered damage, both correct choice and reaction time in the regular network are significantly affected by the distributed damage (Fig. 6 (a), Fig. 6 (b)). For instance, at the damage level $p_{\text {dam }}=0.6$, the regular network shows very poor performance of decision-making, which is indicated by the lower correct choice and longer reaction time. The random network still shows gradually decreased correct choices and gradually increased reaction times when $p_{\text {dam }}$ varies from 0.2 to 0.6 (Fig. 6(c), Fig. 6(d)). Finally, the small-world network also shows gradually changed performance in the decision-making process (Fig. 6(e), Fig. 6(f)). However, further comparison of network behaviors at the level $p_{d a m}=0.6$ exhibits that the small-world network has the highest correct choices and the lowest reaction times at the whole range of coherent levels (Fig. 6(g), Fig. 6(h)). The random network still shows the worst performance of decision-making at the damage level $p_{\text {dam }}=0.6$. These results indicate that the small-world network has the best ability to resist the distributed neuronal damage.

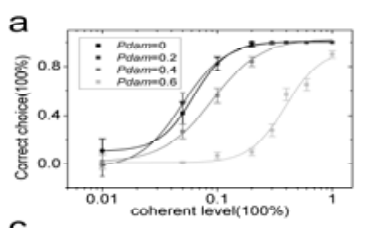

C
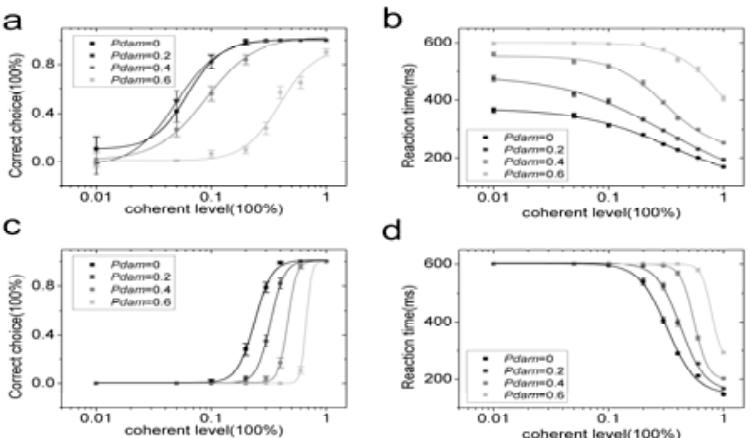

e

d

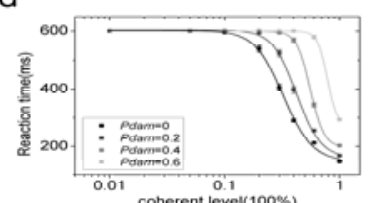

$f$

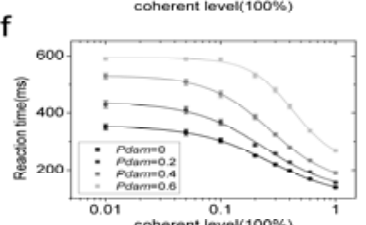

g

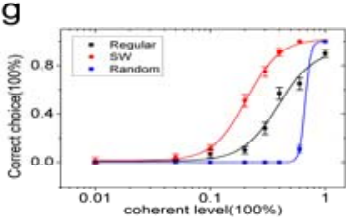

h

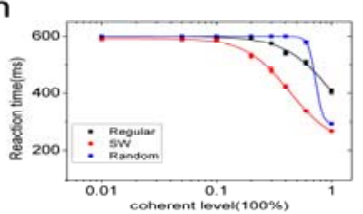

As shown in Figures 6(a) and 6(b), the regular network shows severe decrease of network behaviors in the decision-making process, following gradual increase of distributed neuronal damages. in In Figures 6(c) and 6(d), the random network shows gradually decrease of network behaviors, following gradual increase of neuronal damages. In Figures 6(e) and 6(f), the small-world network shows similar change pattern to the regular network. In Figures $6(\mathrm{~g})$ and $6(\mathrm{~h})$, the comparison of network behaviors $\left(p_{\text {dam }}=0.6\right)$ shows that the small-world network displays the best performance than that in two other networks. The decision-making process is simulated in the low internal noise $(\sigma=0.5)$. Data are fitted by the logistic curve ( $R^{2}>0.98$ for all curves). In each test point the data are obtained after average of 100 trials. The error bar indicates SEM.

The above changes of network behaviors may be due to the corresponding changes of network topology in the case of neuronal damages. Therefore, we calculated the topological features of the networks. As shown in Table 3, the regular network shows different changes of topological features between two different neuronal damage patterns, especially the inward and outward degrees. In the distributed damage pattern, the degrees of the regular network are significantly reduced. However, in the clustered damage pattern, the degrees are slightly decreased. The random network shows similar changes of topological features in both damage patterns (Table 4). Finally, the small-world network shows similar change patterns to those in the regular network. That is, two types of neuronal damages induce different changes of topological features (Table 5). Considering the results of network behaviors, these results indicate that there are strong correlations between the changes of network behaviors and the changes of topological features (See Discussion).

Table 3 Topological features of the regular network under the conditions of neuronal damages. NaN indicates nonsense data because of some infinite values.

\begin{tabular}{cccccccccc}
\hline & \multicolumn{3}{c}{ distributed damage pattern } & \multicolumn{5}{c}{ clustered damage pattern } \\
\cline { 2 - 9 } $\mathrm{P}_{\text {dam }}$ & $\mathrm{K}_{\text {in }}$ & $\mathrm{K}_{\text {out }}$ & $\mathrm{cc}$ & $\mathrm{pl}$ & $\mathrm{K}_{\text {in }}$ & $\mathrm{K}_{\text {out }}$ & $\mathrm{cc}$ & $\mathrm{pl}$ \\
\hline 0 & 20 & 20 & 0.711 & 5.477 & 20 & 20 & 0.711 & 5.477 \\
0.2 & 15.958 & 15.958 & 0.711 & 5.597 & 19.313 & 19.313 & 0.731 & 5.827 \\
0.4 & 11.928 & 11.928 & 0.711 & 5.792 & 19.083 & 19.083 & 0.738 & 4.497 \\
0.6 & 7.862 & 7.862 & 0.709 & $\mathrm{NaN}$ & 18.625 & 18.625 & 0.751 & 3.171 \\
\hline
\end{tabular}

Figure 6 Effects of network topology on the performance of decision-making in the case of the distributed neuronal damage. 
Table 4 Topological features of the random network under the conditions of neuronal damages.

\begin{tabular}{cccccccccc}
\hline & \multicolumn{3}{c}{ distributed damage pattern } & \multicolumn{5}{c}{ clustered damage pattern } \\
\cline { 2 - 9 } $\mathrm{P}_{\text {dam }}$ & $\mathrm{K}_{\text {in }}$ & $\mathrm{K}_{\text {out }}$ & $\mathrm{cc}$ & $\mathrm{pl}$ & $\mathrm{K}_{\text {in }}$ & $\mathrm{K}_{\text {out }}$ & $\mathrm{cc}$ & $\mathrm{pl}$ \\
\hline 0 & 20 & 20 & 0.191 & 2.021 & 20 & 20 & 0.191 & 2.02 \\
0.2 & 15.998 & 15.998 & 0.191 & 2.079 & 15.971 & 15.971 & 0.192 & 2.082 \\
0.4 & 11.875 & 11.875 & 0.191 & 2.178 & 11.925 & 11.925 & 0.191 & 2.173 \\
0.6 & 7.986 & 7.986 & 0.193 & 2.315 & 7.959 & 7.959 & 0.189 & 2.32 \\
\hline
\end{tabular}

Table 5 Topological features of the small-world network under the conditions of neuronal damages. $\mathrm{NaN}$ indicates nonsense data because of some infinite values.

\begin{tabular}{cccccccccc}
\hline & \multicolumn{3}{c}{ distributed damage pattern } & \multicolumn{5}{c}{ clustered damage pattern } \\
\cline { 2 - 9 } dam_p & $\mathrm{K}_{\text {in }}$ & $\mathrm{K}_{\text {out }}$ & $\mathrm{cc}$ & $\mathrm{pl}$ & $\mathrm{K}_{\text {in }}$ & $\mathrm{K}_{\text {out }}$ & $\mathrm{cc}$ & $\mathrm{pl}$ \\
\hline 0 & 20 & 20 & 0.504 & 2.438 & 20 & 20 & 0.502 & 2.435 \\
0.2 & 15.968 & 15.968 & 0.502 & 2.505 & 18.988 & 18.988 & 0.546 & 2.411 \\
0.4 & 11.962 & 11.962 & 0.503 & 2.64 & 18.404 & 18.404 & 0.591 & 2.329 \\
0.6 & 7.926 & 7.926 & 0.498 & $\mathrm{NaN}$ & 17.574 & 17.574 & 0.65 & 2.185 \\
\hline
\end{tabular}

\section{Discussion}

In the present study, we constructed a recurrent network model to simulate the decision-making process. Compared with the previous recurrent model of decision-making (Wang, 2002), here we simplify the dynamical process of neuronal firing by ignoring the detailed descriptions of synaptic currents, such as currents mediated by AMPA, NMDA, and GABA receptors. However, we focus our study on the effects of network topology on the performance of decision-making by constructing three different types of networks: the regular network, the random network, and the small-world network. We found that the networks containing large regular components show better performance of decision-making than the network with random components in the case of low internal noise. However, in the case of high internal noise, the networks with random components show better performance of decision-making. In addition, in the case of neuronal damage, especially largely distributed neuronal damage, the small-world network remains the best ability to execute the decision-making process. All these results indicate that the small-world network, which contains both regular component and random component of network architecture, exhibits the relatively stable network behavior in the alternative decision-making process.

In our model, we didn't examine the effects of the scale-free network (Barabasi \& Albert, 1999), which has been identified in many biological and other systems (Albert \& Barabasi, 2002). That's because we think there is no biological significance to construct a neural group with scale-free topology. The neural groups we constructed are used to mimic a particular brain structure, which contributes to the decision-making process. In a brain structure, there are no biological evidences to support the assumption that a few neurons own the large proportion of synaptic connections. Instead most of neurons inside a brain structure should be homogenous in the aspect of connection probability. Otherwise, removal (in case of neurodegeneration or neural injury) of some "essential" neurons may result in fatal damage to the whole brain structure. However, the existence of these "essential" nodes is one of basic properties of scale-free network. In addition, recent evidences show that the formation of some brain structure, such as the brainstem reticular formation, is a small-world, but not scale-free, network (Humphries et al., 2006). Therefore, the scale-free network is excluded in the present study.

In a recurrent network model, the essential factor to win the competition between alternative choices is to rapidly recruit the activated neurons to exceed the firing threshold (Schall, 2001; Mazurek et al., 2003). Due to the large cluster coefficient in the regular network or the small-world network (Table 2 ), the recruitment in these networks is more efficient than that in the random network. As a matter of fact, the regular network and the small-world network have significantly higher correct choice and lower reaction time than those in the random network in the case of low internal noise. Thus, these results imply that the cluster coefficient may be the key parameter to control the network behavior in the decision-making process when the internal noise is low. However, the high internal noise leads to large variation of neuronal firing rates. Then, the large cluster coefficient in the regular network and the small-world network may also enlarge the error signals of those variations, which results in more wrong output of decision-making. In the other hand, the short path length in the random network may contribute to rapid dispersal of error signals. Therefore, the regular network and the small-world network show lower correct choice than that in the random network when the internal noise is high. However, large cluster coefficients still 
contribute to the rapid recruitment of competing signals no matter these signals are efficient signals or error signals when the firing rates are lower than the threshold. Thus, the regular network and the small-world networks still show significantly lower reaction time than that in the random network, especially in the case of low coherent input levels. When the neuronal states in the stimulated cluster exceed the firing threshold, the essential factor to win the competition is to recruit more neurons outside that cluster. In this case, the short path length in the small-world network helps the spread of the competing signals to the inactivated neurons, which leads to a larger recruitment cluster, compared with the recruitment in the regular network. Therefore, the small-world network shows the lowest reaction time among three networks. Taken together, different network features may contribute to network behaviors in the different conditions.

The robustness of the network behavior is a very important criterion of the network. Many complex systems display a surprising degree of tolerance for errors (Albert et al., 2000; Albert \& Barabasi, 2002). For example, the removal of some nodes only has few effects on the network connections or network behaviors. In the present study, we introduced neuronal damages to examine this error tolerance or attack vulnerability. Different topological networks have totally different connections. For instance, neurons in the regular network prefer to connect adjacent neurons, while neurons in the random network randomly connect other neurons. Therefore, different damage patterns may have different effects on the network behavior. Our results improve this possibility. We found that the distributed damage pattern causes significantly different effects on the performance of decision-making in the regular network, compared with the clustered damage pattern. The clustered damage in the regular network almost has no effects on the network behavior (Fig. 5). However, the distributed damage causes severe reduce of the performance of decision-making in both aspects of correct choice and reaction time (Fig. 6). Statistical results of network topology show that the inward and outward degrees of nodes in the regular network are significantly decreased, following the increase of the distributed damage levels. Meanwhile, the clustered damage only has small effects on the degrees of nodes (Table 3 ). This reduced degrees caused by the distributed damage lead to the decrease of the recruitment of more neurons in the alternative competition, which consequently results in the decrease of the performance of decision-making. For the random network, two damage patterns have similar effects on the topological features (Table 4), and finally cause similar decrease of the network behaviors. For the small-world network, two damage patterns also induce similar changes of topological features and network behaviors to those in the regular network (Table 5). That is, the distributed damage pattern causes more severe decrease of the network behaviors than the clustered damage pattern. However, the changes are quantitatively different between two networks. In the case of clustered damage $p_{\text {dam }}=0.6$, the regular network shows slightly better performances of decision-making than that in the small-world, although the performances in both networks are just varied a little (Fig. 5). However, in the case of distributed damage $p_{\text {dam }}=$ 0.6 , the small-world network shows significantly better performance than the regular network in the both aspects of correct choice and reaction time (Fig. 6). These results indicate that the small-world network has better ability to resist neuronal damages, especially the distributed damage.

Taken together, different topological networks have some advantages in different conditions. In total, the small-world network shows good network behaviors in the cases of high internal noise and neuronal damages. Our findings give a hint that the network topology is one of essential factors to consider during the construction of neural networks. Moreover, our results from the computational model may contribute to the understanding of neuronal connections in the biological brain. In other words, the good performance of the small-world network raises the following question, that is, are the neuronal connections in a particular brain region of animals or human being organized as the small-world pattern?

\section{References}

[1] Albert R \& Barabasi AL. Statistical mechanics of complex networks. Rev Mod Phys 2002, 74, 47-97.

[2] Albert R, Jeong H \& Barabasi AL. Error and attack tolerance of complex networks. Nature 2000, 406, 378-382.

[3] Amit DJ \& Brunel N. Model of global spontaneous activity and local structured activity during delay periods in the cerebral cortex. Cereb Cortex 1997, 7, 237-252.

[4] Barabasi AL \& Albert R. Emergence of scaling in random networks. Science 1999, 286, 509-512.

[5] Boccaletti S, Latora V, Moreno Y, Chavez M \& Hwang DU. Complex networks: Structure and dynamics. Phys Rep 2006, 424, 175-308.

[6] Bogacz R. Optimal decision-making theories: linking neurobiology with behaviour. Trends Cogn Sci 2007, 
$11,118-125$.

[7] Bogacz R, Brown E, Moehlis J, Holmes P \& Cohen JD. The physics of optimal decision making: a formal analysis of models of performance in two-alternative forced-choice tasks. Psychol Rev 2006, 113, 700-765.

[8] Britten KH, Shadlen MN, Newsome WT \& Movshon JA. Responses of neurons in macaque MT to stochastic motion signals. Vis Neurosci 1993, 10, 1157-1169.

[9] Busemeyer JR \& Townsend JT. Decision field theory: a dynamic-cognitive approach to decision making in an uncertain environment. Psychol Rev 1993, 100, 432-459.

[10] Ditterich J. Evidence for time-variant decision making. Eur J Neurosci 2006, 24, 3628-3641.

[11] Durstewitz D, Seamans JK \& Sejnowski TJ. Neurocomputational models of working memory. Nat Neurosci 2000, 3 Suppl, 1184-1191.

[12] Glimcher PW. The neurobiology of visual-saccadic decision making. Annu Rev Neurosci 2003, 26, 133-179.

[13] Hansel D, Mato G, Meunier C \& Neltner L. On numerical simulations of integrate-and-fire neural networks. Neural Comput 1998, 10, 467-483.

[14] Humphries MD, Gurney K \& Prescott TJ. The brainstem reticular formation is a small-world, not scale-free, network. P Roy Soc B-Biol Sci 2006, 273, 503-511.

[15] Mazurek ME, Roitman JD, Ditterich J \& Shadlen MN. A role for neural integrators in perceptual decision making. Cereb Cortex 2003, 13, 1257-1269.

[16] Newman MEJ. The structure and function of complex networks. Siam Rev 2003, 45, 167-256.

[17] Newsome WT, Britten KH \& Movshon JA. Neuronal correlates of a perceptual decision. Nature 1989, 341, 52-54.

[18] Parker AJ \& Newsome WT. Sense and the single neuron: probing the physiology of perception. Annu Rev Neurosci 1998, 21, 227-277.

[19] Platt ML. Neural correlates of decisions. Curr Opin Neurobiol 2002, 12, 141-148.

[20] Roitman JD \& Shadlen MN. Response of neurons in the lateral intraparietal area during a combined visual discrimination reaction time task. J Neurosci 2002, 22, 9475-9489.

[21] Romo R \& Salinas E. Touch and go: decision-making mechanisms in somatosensation. Annu Rev Neurosci 2001, 24, 107-137.

[22] Romo R \& Salinas E. Flutter discrimination: neural codes, perception, memory and decision making. Nat Rev Neurosci 2003, 4, 203-218.

[23] Schall JD. Neural basis of deciding, choosing and acting. Nat Rev Neurosci 2001, 2, 33-42.

[24] Smith PL \& Ratcliff R. Psychology and neurobiology of simple decisions. Trends Neurosci 2004, 27, 161-168.

[25] Sugrue LP, Corrado GS \& Newsome WT. Choosing the greater of two goods: neural currencies for valuation and decision making. Nat Rev Neurosci
2005, 6, 363-375.

[26] Usher M \& McClelland JL. The time course of perceptual choice: the leaky, competing accumulator model. Psychol Rev 2001, 108, 550-592.

[27] Vickers D. Evidence for an accumulator model of psychophysical discrimination. Ergonomics 1970, 13, 37-58.

[28] Wang XJ. Probabilistic decision making by slow reverberation in cortical circuits. Neuron 2002, 36, 955-968.

[29] Watts DJ \& Strogatz SH. Collective dynamics of 'small-world' networks. Nature 1998, 393, 440-442.

[30] Wong KF \& Wang XJ. A recurrent network mechanism of time integration in perceptual decisions. J Neurosci 2006, 26, 1314-1328. 